\title{
Developing personalised integrated psychotherapy for patients with personality disorders
}

\author{
Paul S Links
}

Department of Psychiatry, Schulich School of Medicine \& Dentistry, The University of Western Ontario, Victoria Hospital, North Tower, Rm B8-132, 800 Commissioners Road East, London, Ontario, Canada N6A 5W9; paul.links@lhsc.on.ca

\section{WHAT IS ALREADY KNOWN ON THIS TOPIC?}

Several psychotherapy treatments specifically developed for patients with borderline personality disorder (BPD) have proven to be effective. ${ }^{1}$ General psychiatric management (GPM) is one of the therapies that have been shown to be effective for reducing recurrent suicidal and self-harm behaviour, symptom distress and borderline personality disorder features. ${ }^{2}$ However, these proven effective therapies ${ }^{12}$ have generally been delivered over 1-3 years of therapy.

\section{WHAT THIS PAPER ADDS?}

- Kramer and colleagues have tested the effectiveness of GPM given over only 10 sessions or 3-month duration of the treatment.

- This study attempts to adopt psychotherapeutic 'personalised medicine' by using an individualised relationship intervention as an added therapy ingredient with GPM.

- The approach may also improve the working alliance between patients with borderline personality and their therapists early in the course of therapy.

\section{LIMITATIONS}

- The outcomes were not measured beyond the 3-month duration of the treatment.

- The study outcomes were limited to self-report measures, and suicide and self-harm behaviours were not measured as specific outcomes.

- The therapists providing GPM were much less experienced than the therapists in the original study (2.5 vs 14.2 years of experience).

\section{WHAT NEXT IN RESEARCH}

- Specific trials are needed to test shorter durations of psychotherapies versus standard duration; for example, testing 6 months vs 12 months of GPM is warranted.

- Comparing 'personalised', integrated psychotherapies versus particular, theoretical, model-driven approaches of psychotherapy will be useful to determine if they improve engagement and symptom outcomes.

\section{COULD THESE RESULTS CHANGE YOUR PRACTICES AND WHY?}

No, the current results need to be replicated and shown to have longer term benefits before mental health professionals modify their therapies for patients with BPD. However, there is growing interest in developing shorter duration therapies for patients with BPD. For example, 6 months of dialectical behaviour therapy (DBT) (an abbreviated form of year-long DBT) was shown to be effective for reducing non-suicidal self-injury, suicide ideation, depression and hopelessness. ${ }^{3}$ In addition, highly 'personalised' integrated psychotherapeutic approaches may be more beneficial than adherence to a particular theoretical model of therapy. The theoretical approach suggests that one size fits all rather than attending to the specific patient needs.

Competing interests None.

doi:10.1136/eb-2014-101935

\section{REFERENCES}

1. Zanarini MC. Psychotherapy of borderline personality disorder. Acta Psychiatr Scand 2009;120:373-7.

2. McMain SF, Links PS, Gnam WH, et al. A randomized trial of dialectical behavior therapy versus general psychiatric management for borderline personality disorder. Am J Psychiatry 2009;166:1365-74.

3. Stanley B, Brodsky B, Nelson JD, et al. Brief dialectical behavior therapy (DBT-B) for suicidal behavior and non-suicidal self-injury. Arch Suicide Res 2007:11:337-41.

ABSTRACT FROM: Kramer U, Kolly S, Berthoud L, et al. Effects of motive-oriented therapeutic relationships in a ten-session general psychiatric treatment of borderline personality disorder: a randomized controlled trial. Psychother Psychosom 2014;83:176-86.

Patients/participants Eighty-five adults (mean age 33 years; $69 \%$ female) with Diagnostic and Statistical Manual of Mental Disorders, Fourth Edition (DSM-IV) borderline personality disorder (BPD). People with a DSM-IV diagnosis of psychotic disorder, mental retardation or substance abuse were excluded.

Setting An outpatient university psychiatric clinic in Switzerland; May 2010 to March 2013.

Intervention General psychiatric management (GPM) augmented with plan analysis (PA) and motive-oriented therapeutic relationship (MOTR) therapy $(n=42)$. Both intervention and control conditions involved 10 sessions of GPM delivered over 3 months. This involved a psychiatric and psychotherapeutic approach including establishing treatment focus, short-term goals and improving motivation, and identifying core conflicting themes. In the intervention group PA and MOTR techniques were 'infused' in the process between sessions 2 and 10 .

Comparison GPM alone $(n=43)$.

Patient follow-up Sixty people (81\%) completed, and 74 (87\%) were included in intention-to-treat (ITT) analysis, excluding 11 people who withdrew after the first session and were classed as non-engagers. No difference in drop-out between groups.
Allocation Concealed.

Blinding Single (participant).

\section{OUTCOMES}

Outcome Questionnaire (OQ-45) On ITT analysis using analysis of covariance (ANCOVA) to control for baseline symptom level, MOTR and PA gave a greater overall improvement in total score compared to GPM alone $\left(\mathrm{F}_{1,73}=7.25, \mathrm{p}<0.02\right)$. This remained significant in the completer analyses $\left(\mathrm{F}_{1,59}=5.26, \mathrm{p}=0.02\right)$. Using multivariate ANCOVA (MANCOVA) there was a borderline significant effect favouring MOTR and PA on all three OQ-45 subscales (symptoms, interpersonal and social problems; $\mathrm{F}_{3,67}=2.50, \mathrm{p}<0.06$ ).

Inventory of interpersonal problems There was no significant difference between groups on ITT analysis. MOTR and PA had a borderline significant effect on interpersonal problems compared to GPM alone on completer analyses $\left(\mathrm{F}_{1,50}=3.22, \mathrm{p}=0.07\right)$.

Borderline symptom list (BSL-23) There was no significant difference between groups on either ITT or completer analyses.

Therapeutic alliance A stronger therapeutic alliance as assessed by the therapist developed in the MOTR and PA group compared to GPM alone $\left(Z_{55}=0.99, \mathrm{p}<0.04\right)$. 